\title{
A combination of three common inherited mitochondrial DNA polymorphisms promotes longevity in Finnish and Japanese subjects
}

\author{
Anna-Kaisa Niemi ${ }^{1,2}$, Jukka S Moilanen ${ }^{1,2}$, Masashi Tanaka ${ }^{3}$, Antti Hervonen ${ }^{4}$, \\ Mikko Hurme ${ }^{5}$, Terho Lehtimäki ${ }^{6}$, Yasumichi Arai ${ }^{7}$, Nobuyoshi Hirose ${ }^{7}$ and \\ Kari Majamaa*,1,2
}

\begin{abstract}
${ }^{1}$ Department of Neurology, University of Oulu, Oulu, Finland; ${ }^{2}$ Clinical Research Center, Oulu University Hospital, Oulu, Finland; ${ }^{3}$ Department of Gene Therapy, Gifu International Institute of Biotechnology, Kakamigahara, Japan; ${ }^{4}$ Laboratory of Gerontology, Tampere School of Public Health, University of Tampere, Tampere, Finland; ${ }^{5}$ Department of Microbiology and Immunology, University of Tampere Medical School and Tampere University Hospital, Tampere, Finland; ${ }^{6}$ Department of Clinical Chemistry, Tampere University Hospital, Tampere, Finland; ${ }^{7}$ Department of Geriatric Medicine, Keio University School of Medicine, Tokyo, Japan
\end{abstract}

Mitochondrial DNA (mtDNA) coding region polymorphisms, as well as the 150T polymorphism in the noncoding region, have been associated with longevity. We have studied here the association of 150T with longevity further and assessed differences in this association between various mtDNA haplogroups. We analysed a sample of 321 very old subjects and 489 middle-aged controls from Finland and Japan. 150T was more frequent among the very old than among the controls in both the Finnish and Japanese subjects. Interestingly, the association was not similar in all haplogroups, and a stratified analysis revealed that two additional common polymorphisms, 489C and 10398G, modified the association between 150T and longevity. These findings suggest that longevity is partly determined by epistatic interactions involving these three mtDNA loci. European Journal of Human Genetics (2005) 13, 166-170. doi:10.1038/sj.ejhg.5201308

Published online 13 October 2004

Keywords: mitochondrial DNA; longevity; control region; epistasis; association study; phylogenetic analysis; haplogroup

\section{Introduction}

Mitochondrial DNA (mtDNA) is a maternally inherited genome that encodes 22 tRNAs, two rRNAs and 13 subunits of the respiratory chain complexes and ATP synthase. These complexes catalyse the reactions of oxidative phosphorylation that produce ATP and also contribute to oxygen free radicals, which are thought to play a role in the aging process. ${ }^{1}$ Interestingly, longevity shows maternal

*Correspondence: Professor K Majamaa, Department of Neurology, University of Oulu, PO Box 5000, FIN-90014 Oulu, Finland. Tel: +3588 315 4526; Fax: + 3588315 4544; E-mail: kari.majamaa@oulu.fi Received 6 May 2004; revised 19 August 2004; accepted 1 September 2004 inheritance. ${ }^{2}$ Uniparental inheritance and high mutation rate have led to mtDNA lineages (haplogroups), which are defined by ancient polymorphisms and characterized by considerable variation. The European population is almost exclusively distributed among the nine haplogroups designated as $\mathrm{H}, \mathrm{I}, \mathrm{J}, \mathrm{K}, \mathrm{T}, \mathrm{U}, \mathrm{V}, \mathrm{W}$ and $\mathrm{X}$, whereas haplogroups $\mathrm{A}$, B, C, D, F, G and certain subclusters of macrohaplogroups $\mathrm{M}$ and $\mathrm{N}$ are characteristic to Asian populations, haplogroups A, B, C and D to native Americans and haplogroups L0, L1, L2 and L3 to African populations. ${ }^{3-5}$

There is evidence that certain coding region polymorphisms specific to mtDNA haplogroups may be relevant for common diseases and traits. ${ }^{6}$ Longevity has been found to be associated with mtDNA coding region polymorphisms 
such as 5178A (characterizing haplogroup D) in the Japanese $^{7}$ and 9055A (characterizing haplogroup K) in the French ${ }^{8}$ and Irish, ${ }^{9}$ and mtDNA haplogroup $\mathrm{J}$ in the Italians ${ }^{10}$ and the Finns. ${ }^{11}$ Furthermore, the $150 \mathrm{~T}$ polymorphism within a $1.1 \mathrm{~kb}$ noncoding control region of mtDNA has been reported to be more prevalent in centenarians than in controls. ${ }^{12}$ Interestingly, $150 \mathrm{~T}$ is present in several haplogroups among the global population including haplogroups $\mathrm{D}$ and $\mathrm{J}^{13}$ In this study we identified carriers of $150 \mathrm{~T}$ among 810 very old subjects and middle-aged controls from Finland and Japan in order to examine the association of this polymorphism with longevity further. We also assessed the possibility that the association between 150T and longevity is modified by other control region sequence variation or haplogroupspecific coding region variation.

\section{Subjects and methods}

The group of very old subjects comprised 225 Finns (age 90 or 91 years) and 96 Japanese (age 100-104 years), and the middle-aged controls 393 Finns and 96 Japanese. The controls were from the same geographical regions as the very old subjects. The allele status at position 150 was determined by direct sequencing of the Japanese samples and by allele-specific amplification of the Finnish samples. DNA extracted from blood was amplified in the presence of an oligonucleotide containing an allele-specific Locked Nucleic Acid ${ }^{\circledR}$ (LNA) (Proligo LLC, Paris, France). Samples harbouring $150 \mathrm{C}$ could be amplified in the presence of a forward primer 5'-CTGTCTTTGATTCCTGCCTCATC (LNA underlined), and samples harbouring $150 \mathrm{~T}$ in the presence of a forward primer 5'-CTGTCTTTGATTCCTGCCTCATT. Each sample was amplified in both reactions with $\overline{5^{\prime}}$ CTGTTAAAAGTGCATACCGCCAA as the reverse primer. The amplified 302-bp fragment was visualized by agarose gel electrophoresis. Sequencing of selected samples was used to verify the reliability of the results obtained by allele-specific amplification.

Established haplogroup-defining polymorphisms in the coding region were determined by direct sequencing or by restriction fragment analysis ${ }^{14}$ in the carriers of $150 \mathrm{~T}$ in order to assign the samples to mtDNA haplogroups. ${ }^{3,4}$ The mtDNA control region spanning the nucleotides 1602400576 was sequenced in the samples harbouring 150T.

One-tailed Fisher's exact test was used to assess the hypotheses that the frequency of 150T was higher and the frequencies of haplogroups harbouring 150T were higher among the very old subjects than among the controls. The frequencies of the very old subjects and the controls in each haplogroup were represented as $2 \times 2$ tables, and heterogeneity among these was evaluated by using RelRisk 2.33. ${ }^{15}$ Associations between alleles at polymorphic sites and longevity were measured by the $\chi^{2}$ statistic. An estimate of the significance of the highest observed chi-square $\left(\chi_{\max }^{2}\right)$ was determined in a randomization test, where the labels 'case' and 'control' were permuted 100000 times and the global $P$-value was estimated by the proportion of permutation samples with $\chi_{\max }^{2}$ equal to or higher than that in the observed data.

\section{Results}

We found 65 very old subjects and 66 controls with $150 \mathrm{~T}$ (Table 1), implying a significantly higher frequency among the very old than among the controls in both the Finns and the Japanese. The control region sequence was available for all 150T carriers, except for one U5 control subject who was therefore excluded from subsequent analyses. A total of 88 polymorphic sites were identified, and the strongest association to longevity among these control region sequences was found for 489C $\left(\chi^{2}=14.099\right.$; permutation test: $P=0.00165)$. When the position 489 was excluded, a permutation test revealed that no other control region variants were significantly associated with longevity among the 150 T carriers $\left(\chi_{\max }^{2}=7.075\right.$; permutation test: $\left.P=0.126\right)$. A phylogenetic analysis then revealed that $150 \mathrm{~T}$ and $489 \mathrm{C}$ were both present in subhaplogroups J2, D5 and M7b, whereas 150T, but not 489C, was present in T2, U5 and N9a (Figure 1). In addition, the coding region polymorphism 10398G was found to co-segregate with 489C.

A contingency table analysis of the frequencies of the very old subjects and the controls in subhaplogroups J2, D5, M7b, T2, U5 and N9a revealed a significant heterogeneity $\left(\chi^{2}=17.604, \mathrm{df}=5, P=0.0035\right)$. Subhaplogroups $\mathrm{J} 2$, D5 and M7b were more common among the very old, and the combined frequency of these subhaplogroups was significantly higher among the very old subjects than among the controls (Table 1).

\section{Discussion}

Secondary origins of heavy strand replication of mtDNA (nucleotide positions 146-151) are located in the vicinity of nucleotide position 150, and it has been suggested that 150T alters the location of the origin of heavy strand replication and that $150 \mathrm{~T}$ is related to the regulation of mtDNA replication, ${ }^{12}$ for instance by providing a replicative advantage to a genome that harbours the mutation. The proportion of $150 \mathrm{~T}$ relative to $150 \mathrm{C}$ in fibroblasts has been found to increase with advanced age. ${ }^{12}$ Other polymorphisms in this region could have a similar effect, and $152 \mathrm{~T}>\mathrm{C}$ and $195 \mathrm{~T}>\mathrm{C}$ have also been shown to accumulate in the fibroblasts of aged individuals ${ }^{16}$ and $189 \mathrm{~A}>\mathrm{G}$ in the muscle. ${ }^{17}$ We found that the majority of the samples belonging to J2 harboured at least four mutations close to the origins of replication of mtDNA, whereas similar pattern was not found for D5, M7b, N9a, $\mathrm{T} 2$, or U5. Therefore, polymorphisms near the origin of the 
Table 1 Frequency of subjects with the 150T allele among the very old subjects and middle-aged controls

\begin{tabular}{|c|c|c|c|c|c|}
\hline & \multicolumn{2}{|c|}{ Very old } & \multicolumn{2}{|c|}{ Controls } & \multirow[b]{2}{*}{ P-value } \\
\hline & $N$ & $\%$ & $N$ & $\%$ & \\
\hline \multicolumn{6}{|l|}{ Finns and Japanese } \\
\hline All haplogroups & 65 & 20.2 & 66 & 13.5 & $0.007^{*}$ \\
\hline Subhaplogroups $12, \mathrm{D} 5, \mathrm{M} 7 \mathrm{~b}$ & 22 & 6.9 & 5 & 1.0 & $7.8 \times 10^{-6 *}$ \\
\hline Subhaplogroups T2, U5, N9a & 37 & 11.5 & 56 & 11.5 & 0.53 \\
\hline Other haplogroups & 6 & 1.8 & 5 & 1.0 & 0.24 \\
\hline \multicolumn{6}{|l|}{ Finns } \\
\hline All haplogroups & 46 & 20.4 & 57 & 14.5 & $0.037^{*}$ \\
\hline Subhaplogroup J2 & 8 & 3.6 & 3 & 0.8 & $0.015^{\star}$ \\
\hline Subhaplogroup T2 & 1 & 0.4 & 4 & 1.0 & 0.90 \\
\hline Subhaplogroup U5 & 34 & 15.1 & 48 & 12.2 & 0.18 \\
\hline Other haplogroups & 3 & 1.3 & 2 & 0.5 & 0.26 \\
\hline \multicolumn{6}{|l|}{ Japanese } \\
\hline All haplogroups & 19 & 19.8 & 9 & 9.4 & $0.032^{*}$ \\
\hline Subhaplogroup D5 & 7 & 7.3 & 1 & 1.0 & $0.032^{*}$ \\
\hline Subhaplogroup M7b & 7 & 7.3 & 1 & 1.0 & $0.032^{*}$ \\
\hline Subhaplogroup N9a & 2 & 2.1 & 4 & 4.2 & 0.89 \\
\hline Other haplogroups & 3 & 3.1 & 3 & 3.1 & 0.66 \\
\hline
\end{tabular}

Other haplogroups include G, M7a and B4 among very old Japanese subjects, G, B4 and D4 among the Japanese controls, $\mathrm{H}$ in two very old Finnish subjects, an undefined haplogroup in one very old Finnish subject and $\mathrm{H}$ and $\mathrm{U}$ in the Finnish controls. An asterisk denotes significance at the $95 \%$ confidence level $(P<0.05)$, unadjusted for multiple comparisons.

heavy strand replication could explain the association between longevity and subhaplogroup J2, but not the association between longevity and D5 and M7b.

$150 \mathrm{C}>\mathrm{T}$ polymorphism emerged separately in the early evolution of the European subhaplogroups J2, T2 and U5, and of the Asian subhaplogroups D5, M7b and N9a, but has only occasionally been noted elsewhere in the mtDNA phylogeny. Subhaplogroups D5 and M7b of the Japanese belong to mtDNA macrohaplogroup $M$, which has diverged from African haplogroup L3 and from macrohaplogroup $\mathrm{N}$ some 60000 years ago. ${ }^{18}$ On the other hand, N9a of the Japanese and J2, T2 and U5 of the Finns belong to macrohaplogroup $\mathrm{N}$. Most of the haplogroups in macrohaplogroup $\mathrm{N}$ harbour an ancient 10398G $>$ A mutation, which alters the amino acid 114 in the MTND3 gene, but haplogroup $\mathrm{J}$ has experienced a back-mutation at this site resulting in the $10398 \mathrm{G}$ allele in common with macrohaplogroup $\mathrm{M}$ and, therefore, common with D5 and M7b. In addition, haplogroup J harbours the control region mutation $489 \mathrm{~T}>\mathrm{C}$, which also occurred early in the evolution of macrohaplogroup M. Our data thus showed that $150 \mathrm{~T}$ is associated with longevity in subhaplogroups
J2, D5 and M7b that harbour 10398G and 489C, but not in subhaplogroups T2, U5 and N9a that lack the latter two polymorphisms. The association between a combination of these three mtDNA variants and longevity provides the first epidemiological support for the assumption that the pathogenic or adaptive nature of a variant is influenced by interactions with other loci in mtDNA. ${ }^{19}$

Our previous study on Finnish nonagenarians has suggested an association between haplogroups $\mathrm{J}$ and $\mathrm{U}$ and longevity, respectively, and a negative association between haplogroup $\mathrm{H}$ and longevity. ${ }^{11}$ The absence of 150T in haplogroup $\mathrm{H}$ coincides with the lower frequency of this haplogroup among the very old and, similarly, the higher frequency of haplogroup J among the very old coincides with the presence of 150T within haplogroup J2. However, there is discrepancy between the higher frequency of haplogroup $U$ among the very old and the lack of association between 150T and longevity within subhaplogroup U5, suggesting that other yet unidentified polymorphisms in mtDNA contribute to the association between haplogroup $U$ and longevity.

Longevity is a complex trait in which variation depends on epistatic interactions between several genetic loci and

Figure 1 Phylogenetic tree depicting the origin and evolution of mtDNA subhaplogroups harbouring 150C $>$ T (M7b, D5, N9a, U5, T2 and J2). Polymorphisms detected in mtDNA control region spanning nucleotides 16024-00576 in the very old subjects and the controls are shown in normal font, and established haplogroup-defining polymorphisms ${ }^{3,4}$ are shown in italics. Letter suffixes indicate transversions. Insertions, deletions and repeat length polymorphisms are shown according to the recommended nomenclature. @= back-mutation; outgroup= the African reference sequence for human mtDNA (Genbank NC_001807.4, Gl:17981852). The root of the phylogeny includes the transition at position 150, because the outgroup sequence coincidentally contains a parallel 150T allele. Each solid circle represents one very old subject and each open circle represents a control subject. 


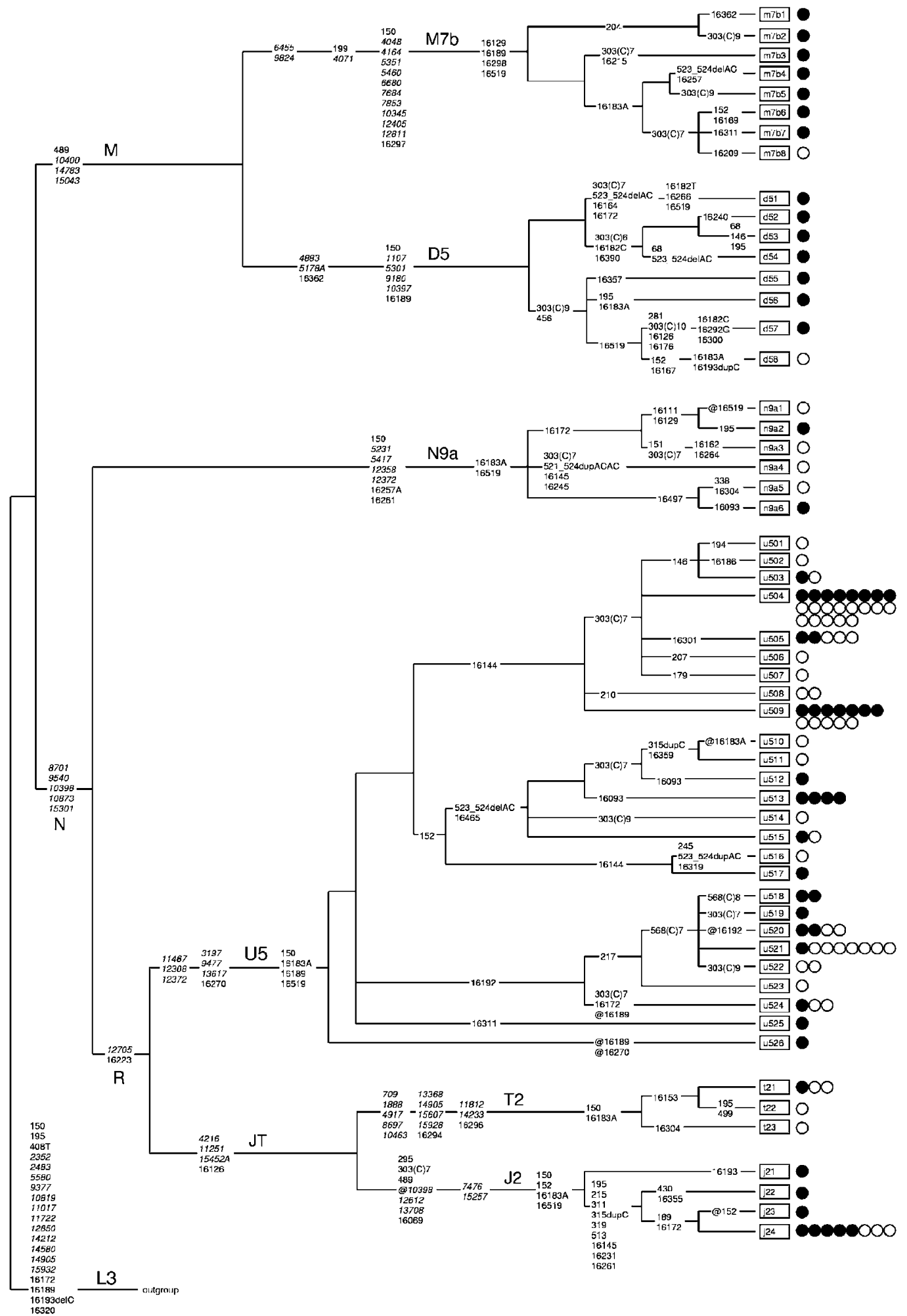


on environmental exposures during life. ${ }^{20}$ Previous analyses of association between mtDNA variation and longevity $^{7,8}$ have focused on single loci and not taken into account nucleotide variation elsewhere in the genome. If several loci play a greater role in determining trait variability than do single loci, a combination of alleles may show a stronger association with a trait than any single locus. This is what we found here for the combination of the 150T, 489C and 10398G alleles, suggesting that mtDNA variation promotes longevity through epistatic effects. The possibility of epistasis also implies that attempts to assess the role of mtDNA variation in complex diseases or traits by single-loci association analyses may overlook loci whose contribution is revealed only when considered in combination with others.

\section{Acknowledgements}

We thank Ms Anja Heikkinen and Ms Pirjo Keränen for their expert technical assistance. This study was supported by grants from the Sigrid Juselius Foundation and the Medical Research Council of the Academy of Finland.

\section{References}

1 Droge W: Oxidative stress and aging. Adv Exp Med Biol 2003; 543: $191-200$

2 Brand FN, Kiely DK, Kannel WB, Myers RH: Family patterns of coronary heart disease mortality: The Framingham Longevity Study. J Clin Epidemiol 1992; 45: 169-174.

3 Herrnstadt C, Elson JL, Fahy E et al: Reduced-median-network analysis of complete mitochondrial DNA coding-region sequences for the major African, Asian, and European haplogroups. Am J Hum Genet 2002; 70: 1152-1171.

4 Kong QP, Yao TG, Sun C, Bandelt HJ, Zhu CL, Zhang YP: Phylogeny of East Asian mitochondrial lineages inferred from complete sequences. Am J Hum Genet 2003; 73: 671-676.

5 Silva Jr WA, Bonatto SL, Holanda AJ et al: Mitochondrial genome diversity of Native Americans supports a single early entry of founder populations into America. Am J Hum Genet 2002; 71: 187-192.
6 Ruiz-Pesini E, Mishmar D, Brandon M, Procaccio V, Wallace DC: Effects of purifying and adaptive selection on regional variation in human mtDNA. Science 2004; 303: 223-226.

7 Tanaka M, Gong J-S, Zhang J, Yoneda M, Yagi K: Mitochondrial genotype associated with longevity. Lancet 1998; 351: 185-188.

8 Ivanova R, Leapage V, Charron D, Schächter F: Mitochondrial genotype associated with French Caucasian centenarians. Gerontology 1998; 44: 349.

9 Ross OA, McCormack R, Curran MD et al: Mitochondrial DNA polymorphism: its role in longevity of the Irish population. Exp Gerontol 2001; 36: 1161-1178.

10 De Benedictis G, Rose G, Carrieri G et al: Mitochondrial DNA inherited variants are associated with successful aging and longevity in humans. FASEB J 1999; 13: 1532-1536.

11 Niemi AK, Hervonen A, Hurme M, Jylhä M, Karhunen PJ, Majamaa K: Mitochondrial DNA polymorphisms associated with longevity in a Finnish population. Hum Genet 2003; 112: 29-33.

12 Zhang J, Asin-Cayuela J, Fish J et al: Strikingly higher frequency in centenarians and twins of mtDNA mutation causing remodeling of replication origin in leucocytes. Proc Natl Acad Sci USA 2003; 100: $1116-1121$.

13 Coskun PE, Ruiz-Pesini E, Wallace DC: Control region mtDNA variants: longevity, climatic adaptation, and a forensic conundrum. Proc Natl Acad Sci USA 2003; 100: 2174-2176.

14 Finnilä S, Lehtonen MS, Majamaa K: Phylogenetic network for European mtDNA. Am J Hum Genet 2001; 68: 1475-1484.

15 Ott J: Analysis of human genetic linkage, 3rd edn. Baltimore: The Johns Hopkins University Press, 1999.

16 Michikawa Y, Mazzucchelli F, Bresolin N, Scarlato G, Attardi G: Aging-dependent large accumulation of point mutations in the human mtDNA control region for replication. Science 1999; 286: 774-779.

17 Wang Y, Michikawa Y, Mallidis C et al: Muscle-specific mutations accumulate with aging in critical human mtDNA control sites for replication. Proc Natl Acad Sci USA 2001; 98: 4022-4027.

18 Quintana-Murci L, Semino O, Bandelt H-J, Passarino G, McElreavey, Santachiara-Benerecetti A: Genetic evidence of an early exit of Homo sapiens sapiens from Africa through eastern Africa. Nat Genet 1999; 23: 437-441.

19 Moilanen JS, Finnilä S, Majamaa K: Lineage-specific selection in human mtDNA: lack of polymorphisms in a segment of MTND5 gene in haplogroup J. Mol Biol Evol 2003; 20: 2132-2142.

20 Perls T: Genetic and environmental influences on exceptional longevity and the AGE nomogram. Ann NY Acad Sci 2002; 959: $1-13$. 